\title{
A slow coronal mass ejection with rising X-ray source
}

\author{
C. P. Goff ${ }^{1}$, L. van Driel-Gesztelyi ${ }^{1,2,3}$, L. K. Harra $^{1}$, S. A. Matthews ${ }^{1}$, and C. H. Mandrini ${ }^{4}$ \\ 1 Mullard Space Science Laboratory, UCL, Holmbury St. Mary, Dorking, Surrey, RH5 6NT, UK \\ e-mail: cpg1@mssl.ucl.ac.uk \\ 2 Observatoire de Paris, LESIA, FRE 2461 (CNRS), 92195 Meudon Cedex, France \\ 3 Konkoly Observatory, PO Box 67, 1525 Budapest, Hungary \\ 4 Instituto de Astronomia y Fisica del Espacio, CONICET-UBA, CC. 67, Suc. 28, 1428 Buenos Aires, Argentina
}

Received 5 November 2004 / Accepted 6 January 2005

\begin{abstract}
An eruptive event, which occurred on 16th April 2002, is discussed. Using images from the Transition Region and Coronal Explorer (TRACE) at $195 \AA$, we observe a lifting flux rope which gives rise to a slow coronal mass ejection (CME). There are supporting velocity observations from the Coronal Diagnostic Spectrometer (CDS) on the Solar and Heliospheric Observatory $(\mathrm{SOHO})$, which illustrate the helical nature of the structure. Additionally a rising coronal hard X-ray source, which is observed with the Reuven Ramaty High Energy Solar Spectroscopic Imager (RHESSI), is shown to follow the flux rope with a speed of $\sim 60 \mathrm{~km} \mathrm{~s}^{-1}$. It is also sampled by the CDS slit, although it has no signature in the Fe XIX band. Following the passage of this source, there is evidence from the CDS for down-flowing (cooling) material along newly reconnected loops through Doppler velocity observations, combined with magnetic field modeling. Later, a slow CME is observed with the Large Angle and Spectroscopic Coronagraph (LASCO). We combine a height-time profile of the flux rope at lower altitudes with the slow CME. The rising flux rope speeds up by a factor of 1.7 at the start of the impulsive energy release and goes through further acceleration before reaching 1.5 solar radii. These observations support classical CME scenarios in which the eruption of a filament precedes flaring activity. Cusped flare loops are observed following the erupting flux rope and their altitude increases with time. In addition we find RHESSI sources both below and above the probable location of the reconnection region.
\end{abstract}

Key words. Sun: X-ray: gamma rays - Sun: flares - Sun: magnetic fields - Sun: coronal mass ejections (CMEs) Sun: corona - Sun: atmosphere

\section{Introduction}

The relationship between flares and CMEs has been debated for some time, although it is becoming apparent that they may arise from the same magnetic phenomenon. Several driving mechanisms have been proposed, reviews of which can be found in, for example, Klimchuk (2000), Forbes (2000) and Forbes (2003). They fall into two main categories: storage and release; and directly driven scenarios. The most common category, but not universally accepted, is the storage and release scenario. Here a slow build-up of magnetic stress precedes the onset of some kind of instability. The CME starts with the rise of an active region filament (and its overlaying arcade) due to an eruptive instability. Then, below the rising filament as the stretched field lines start to reconnect, an impulsive energy release occurs and is accompanied by the acceleration of the filament eruption. In the main flare phase continuing reconnection forms hot X-ray loops and $\mathrm{H} \alpha$ ribbons at their footpoints, where the particles accelerated during the reconnection process impact the chromosphere. As field lines increasingly distant from the magnetic inversion line reconnect, the reconnection region rises. Therefore the resulting X-ray loop footpoints and the $\mathrm{H} \alpha$ ribbons move apart. The initial instability may be triggered by (i) a twisted flux tube, where the twist or height of the filament is too great; or (ii) a sheared magnetic arcade, in which the magnetic energy built up by shearing motions until a critical value is reached.

Most CME studies are based on coronagraph data, which are limited to observations above a certain altitude. By the time the CME has reached the point of detection, it has normally finished accelerating and continues at a constant velocity (St. Cyr et al. 1997). Regardless of the mechanism, the plasma and associated magnetic field are accelerated from the low atmosphere at velocities that can reach in excess of $1000 \mathrm{~km} \mathrm{~s}^{-1}$. The event presented here is significantly slower and therefore observations of the acceleration profile of the $\mathrm{CME}$, before it reaches the coronagraph field of view, can provide valuable insights into the underlying process.

It is sometimes possible to detect a rising flux rope or filament in the low corona with TRACE (Handy et al. 1999) observations. An example of this can be seen in Gallagher et al. (2003). They demonstrate that a discontinuity is observed when height-time information from the low corona is combined with the more traditional CME measurement in the Large Angle and Spectroscopic Coronagraph (LASCO; Brueckner et al. 1995) 
data. Typically height-time measurements from LASCO can be fitted linearly, demonstrating that the CME has reached a steady velocity. Few observations of the acceleration phase have previously been made. This 21st April 2002 event was reported to have a low initial velocity through a combination of observations from TRACE along with an additional data point from the Ultraviolet Coronagraph Spectrometer (UVCS; Kohl et al. 1995). For its initial height-time behaviour to connect smoothly with the coronagraph observations, an exponential increase in acceleration was required.

Typically below a lifting flux rope/CME there is a complex array of rapid loop and footpoint brightenings, plasma jets and other types of ejecta (e.g. Shibata et al. 1995). A moving high-temperature (15 MK) source has been reported by Tsuneta (1997) in a 2nd December 1991 limb flare. This plasmoid formed during the impulsive phase, above the x-point, in 2 dimensions or more general the $\mathrm{x}$-line in 3 dimensions, and rose with a velocity of $\sim 96 \mathrm{~km} \mathrm{~s}^{-1}$. It is understood that the $\mathrm{x}$-line represents the presumed magnetic reconnection region and below this are $\mathrm{V}$-shaped high temperature ridges representing the newly reconnected loops. A hard X-ray (HXR) source is located at the top of these soft X-ray (SXR) loops; caused by the fast shock acting on the newly reconnected loops. Due to the symmetrical nature of the reconnection, outflows must occur both upward as well as downward and it is believed that the upward flows are responsible for the plasmoid.

The event we present was part of a homologous series first analysed by Veronig \& Brown (2004) and Sui et al. (2004). They found that three homologous flares occurred between 14th April and 16th April 2002. This paper concentrates on the 16th April event. Sui et al. (2004) focused on the RHESSI (Lin et al. 2002) data and identified an associated CME in the LASCO catalogue for their April 15th event. We will demonstrate that there was also an associated CME with the 16th April event. We analyse an extensive array of data and will use this to investigate the acceleration of the CME, as well as the coronal hard X-ray source.

\section{16th April 2002 event}

The flare on 16th April 2002 occurred at 12:52 UT in the northwest quadrant of the Sun (NOAA Active Region 9901). From the Geostationary Operational Environmental Satellite (GOES) soft X-ray light curve (Fig. 1) we see that the M2.5 flare lasted for three hours. Fortunately many instruments observed this event over a broad wavelength range, thus allowing for a comprehensive study.

\subsection{Observations of a rising flux rope}

The event from the 16th April 2002 was observed in the TRACE $195 \AA$ band for the entire duration of the flare (Fig. 2) with a $17 \mathrm{~s}$ cadence and an image scale of $0.5^{\prime \prime}$ per pixel. Standard image corrections were first applied before the pointing offsets were corrected using the methods described in Gallagher et al. (2003). At approximately 12:55 UT, the end of an arcade starts to brighten as the loops begin to fill with hot plasma. These loops appear to grow in height as the

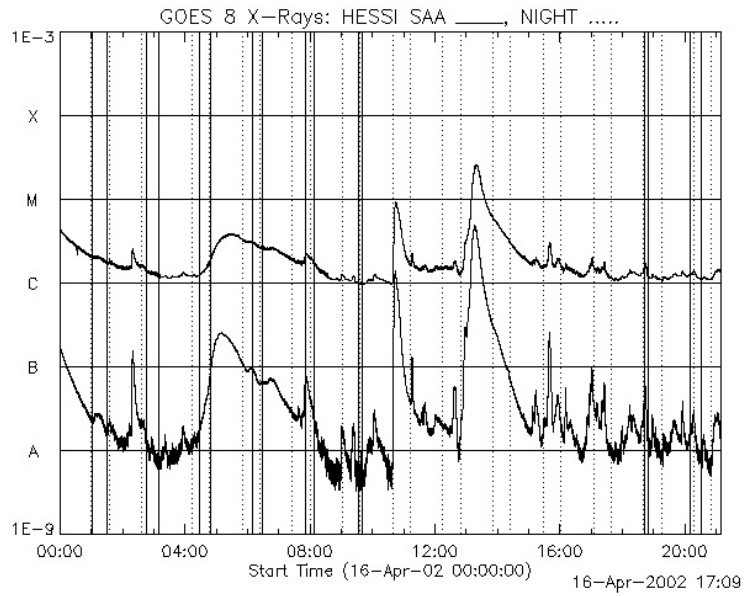

Fig. 1. The GOES light curve for the 16th April 2002. The event studied in this paper occurred at around 12:50 UT.

presumed reconnection continues, and a cusp feature forms at around 13:10 UT. When the region above the bright loops is examined more closely it is possible to see a flux rope, which began rising at about 12:54 UT. This can be seen clearly for $\sim 20 \mathrm{~min}$ and was easily tracked.

Figure 3 shows the rising flux rope more clearly. It is likely to contain heated filamentary material. This will allow the filament material to be seen as emitting for a short time before cooling again. With the identification of a common feature a height-time profile can be extracted from the TRACE data cube. This is achieved by tracking the leading edge of the feature by visual inspection. This process was repeated five times to achieve a consistency, with an error of \pm 5 pixels. The resulting height-time plot for the rising flux rope can be seen in the top panel of Fig. 5.

Initially it was assumed that the height-time plot could be represented with two linear fits. One was calculated for the first nine minutes, the other covered the remainder of the observations. There is a small data gap following the first fit after which the flux rope appears to have increased its velocity. At first the flux rope moves with a constant velocity of $\sim 45 \mathrm{~km} \mathrm{~s}^{-1}$, which was determined from the gradient of the fit with an acceptable $R^{2}$ statistic at 0.89 . The second linear fit indicates an increase in the velocity to some $75 \mathrm{~km} \mathrm{~s}^{-1}$, where the fit had an $R^{2}$ statistic of 0.99 . However, it is not possible to obtain a good linear fit for the whole time period.

In addition there is CDS (Harrison et al. 1995) data for this event. The CDS slit was above the limb at $x=945^{\prime \prime}$ and centred at $y=400 "$ (Fig. 4). The instrument was in a "sit and stare" mode using the $2 \times 240^{\prime \prime}$ slit while observing in Fe XIX. A simple stack plot of intensity slices against time is also presented in Fig. 4. It is clear that just after 13:00 UT there is a rising loop or filament, which is viewed by the slit and seen as a crescent shape in this figure. This crescent is the mirror image of the rising loop, because the tip passes through the slit first followed by the legs. The timing is consistent with that expected from observations in the low corona and can be seen as a cross in Fig. 5. By studying the Doppler velocities of this feature we can see that the bottom left section of the data, which represents 

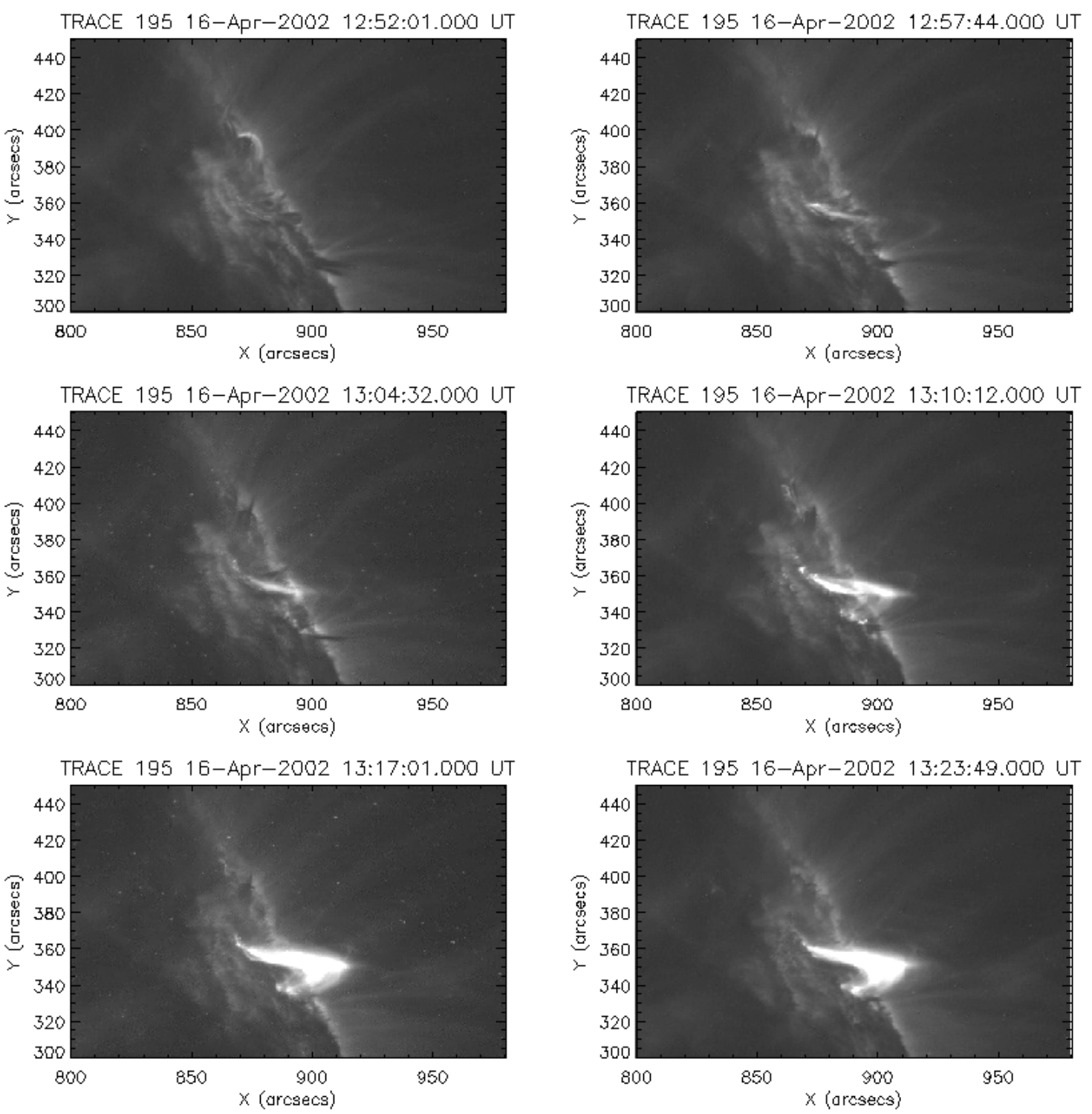

Fig. 2. A time series of TRACE $195 \AA$ A images. It clearly shows the formation of the flaring arcade with the formation of a cusp towards the end.

its leading edge, has a blue shift whereas the rest of the loop structure is red shifted. This is suggestive of helical flows such as those described by Pike \& Mason (2002). Anti-parallel flows along threads forming the filament body may result in a similar picture. However, we recall that erupting filaments are frequently observed to show helical whirling motions. Therefore we believe that the organised pattern of blue-shift followed by red-shift indicates helical flows and is suggestive of a flux rope structure for this erupting filament.

\subsection{RHESSI observations of a rising coronal source}

RHESSI observations provide an additional dataset for this event. Light curves for the flare in the 3-6, 6-12, 12-25 and 25-50 keV energy channels have been constructed, but above $50 \mathrm{keV}$ there is almost no emission. The light curves show a peak around 12:57 UT in the 3-6, 6-12 and the 12-25 keV channels with a much larger, smooth light curve beginning at 13:00 UT and peaking around 13:13 UT in the $25-50 \mathrm{keV}$ band. The PIXON method (Metcalf et al. 1996) was used to reconstruct images, a sample of which can be seen in Fig. 6 . The images were constructed over 5 rotation periods (approximately $20 \mathrm{~s}$ ) in $5 \mathrm{keV}$ bins. Grids 3-9 were used in the processing giving an angular resolution of $\sim 7 "$. General agreement with the configuration illustrated in both Veronig \& Brown (2004) (for the event on the 15th April 2002) and Sui et al. (2004) is shown. The most intense emission comes from the loop top source, which Veronig \& Brown (2004) describe as a loop so dense as to be collisionally thick at electron energies up to $50 \mathrm{keV}$.

In most cases the majority of the emission is located at the dense footpoints of flaring loops. In all cases of this homologous series, of which this flare is a part, the loop itself acts as a dense target. In addition there is another source located above the loop top source (a coronal source); also observed by Sui et al. (2004) in the 16th April event. This is clearly identified in images reconstructed with the PIXON method over $5 \mathrm{keV}$ wide energy bins, in the 5-10, 10-15 and 15-20 keV bands. 

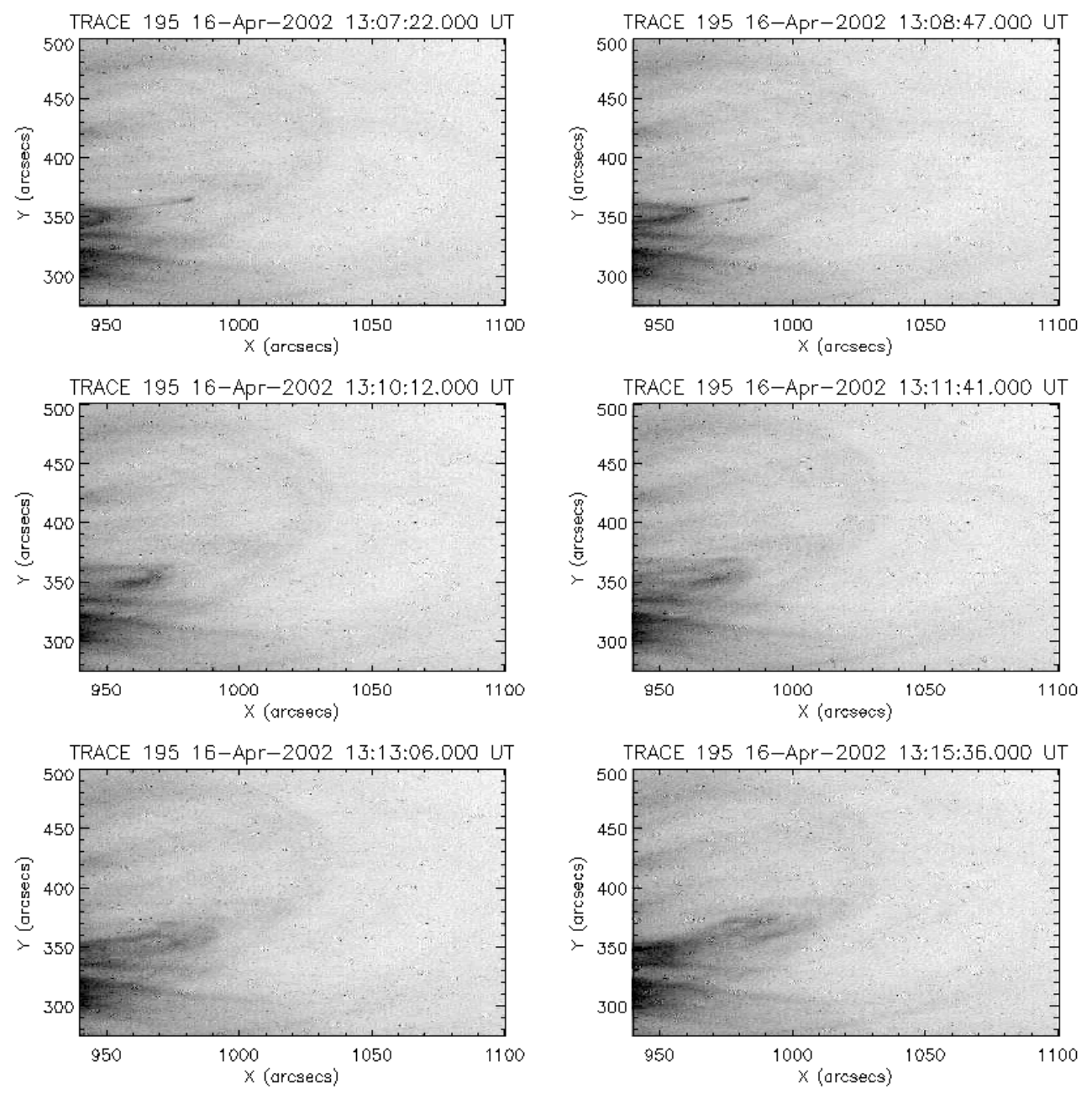

Fig. 3. TRACE $195 \AA$ A images, with a reverse colour table. The field of view has been reduced to give a much clearer view of the lifting flux rope. The images are a time series from top left to bottom right. The lifting flux rope is seen as the dark feature expanding towards the right.
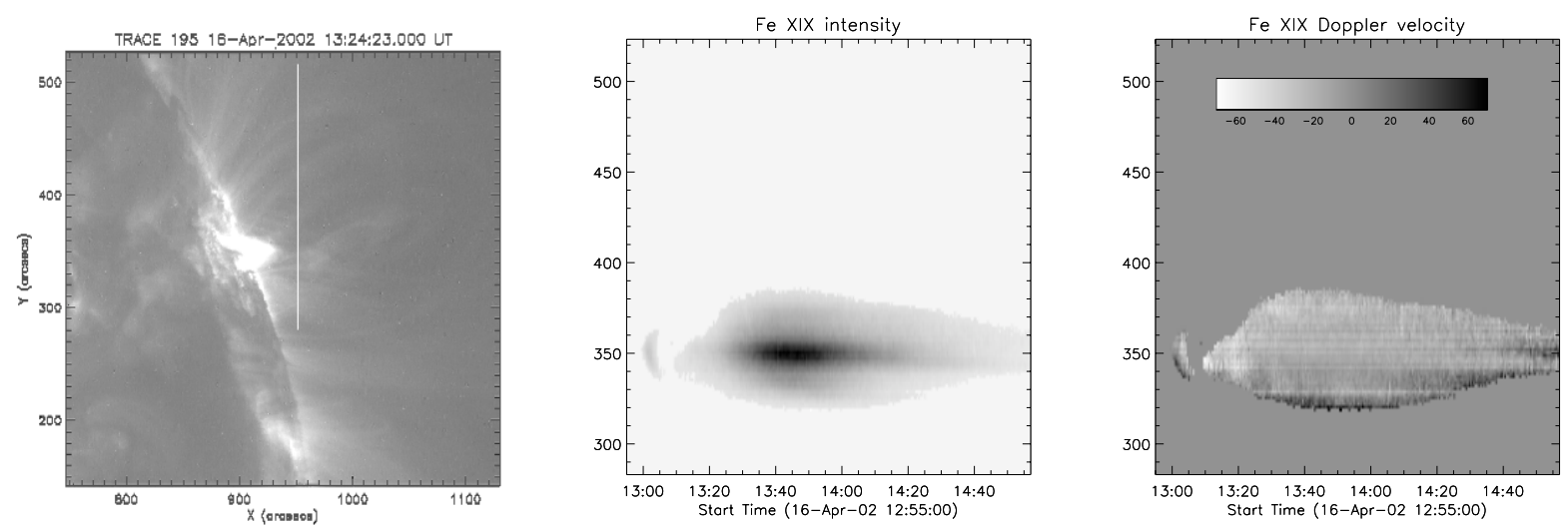

Fig. 4. Left: TRACE $195 \AA$ A image with a line illustrating the position of the CDS slit. Centre: CDS time series of slit intensity images. CDS was in a "sit and stare" mode and this image shows what happened beneath the slit over the time of event. The crescent shape to the left of this image represents the lifting flux rope. Right: here we see the Dopplergram of the same data. The colour bar indicates the Doppler velocity where white is blue-shifted and black is red-shifted.

Note that the dynamic range of RHESSI images is considered to be 10:1, thus all measurements are limited to regions where the brightness is more than one tenth of the peak image brightness at any given time. Although this source was observed by Sui et al. (2004). They reported the source as stationary. However, motion is clearly seen in the height-time plot shown in Fig. 7. This source is weak relative to the bright emission from the loop top and often appears to break up. However the position remains consistent with an outward moving source where the brightest point from the collection of broken sources is used in 


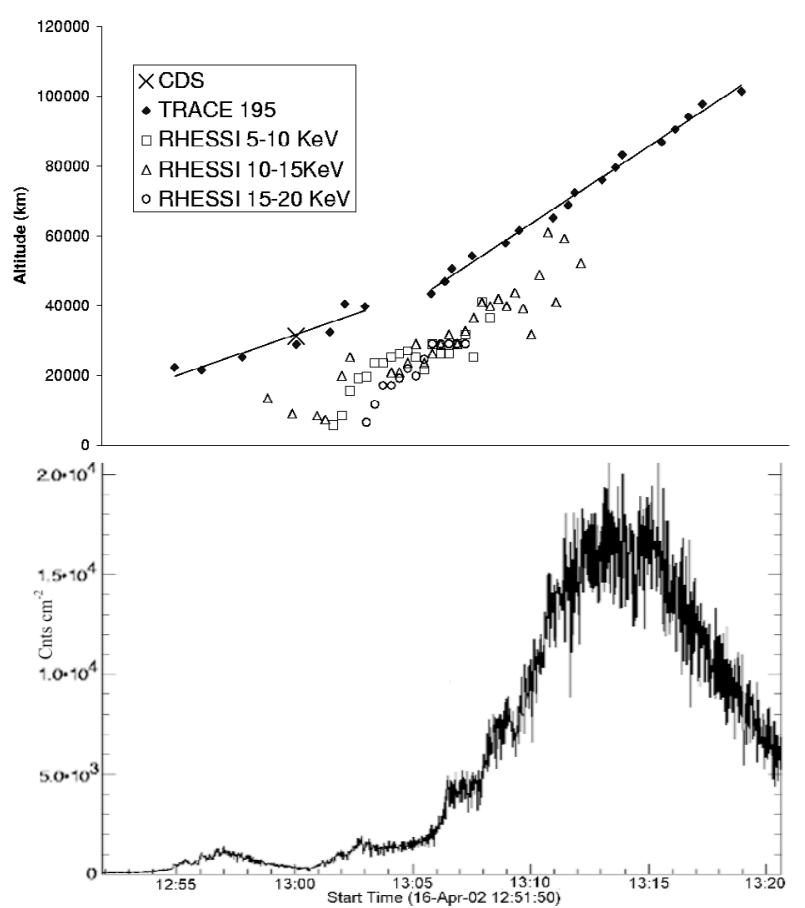

Fig. 5. Top: height-time plot for the lifting flux rope as seen by TRACE. The cross represents the leading edge of the flux rope as seen by CDS. Below the best-fit lines are the data points from the coronal hard X-ray source. Bottom: the hard X-ray light curve including all data between 5 and $50 \mathrm{keV}$ over the same time frame as the height-time plot above.

the plot. It is clear the velocity of this source, at $\sim 60 \mathrm{~km} \mathrm{~s}^{-1}$, is comparable to that of the flux rope at the same point in time. The velocity of the flux rope, from the linear fitting performed earlier, is between 45 and $75 \mathrm{~km} \mathrm{~s}^{-1}$. This is suggestive of an intimate link between the two phenomena.

The coronal source appears weak from the light curves of the different regions. We have included in this paper the light curves for the coronal source in the $10-15 \mathrm{keV}$ band and also for the loop top and footpoint sources (i.e. the whole loop) in the same energy range (Fig. 8). The light curve for the coronal source was constructed by taking a large box around the source. To avoid contamination from the loop-top source the sampling area was moved with the source evolution. Therefore, it was also necessary to move a large sampling area with the loop, again to avoid contamination. One can see immediately that the maximum intensity in the coronal source is almost an order of magnitude below that of the peak in the loop top and footpoint light curve. The emission is gradual and not very impulsive in both the loop top/footpoint source and the coronal source, although the coronal source light curve is much more noisy.

This moving coronal source was viewed by the CDS slit, which had previously observed the rising flux rope. However, at this time there is little, if any, emission seen by CDS in Fe XIX.

\subsection{Cooling down-flows}

Continuing with the CDS observations shown in Fig. 4, we find that the helical structure in the rising flux rope is followed by a gap then by a bright source appears at 13:14 UT. Only data above a reliable threshold is shown. The source expands in size to a maximum of $\sim 65^{\prime \prime}$ at around 13:35 UT and then gradually decreases. This is consistent with the results seen in a separate dataset formed from TRACE. If we take the image cube and produce a synthetic slit at the same position as that of CDS and then produce stack plots, consisting of slices of the image over a time range we get the TRACE equivalent of a CDS image, as shown in Fig. 9. This image shows that the lifting flux rope crosses the slit as in the CDS image with one difference; the emission is from much cooler plasma. The legs of this flux rope can also be seen and they extend much further than in the CDS image. There is weak emission under the flux rope but at around 13:14 the image becomes bright again. This compares well to the emission in CDS. This emission observed by TRACE is largely due to the newly reconnected loops getting higher with time and entering the field of view, although clear loops are not seen passing through the position of the slit in the TRACE movies. This is probably an effect of dynamic range plotted at any one time, but the stack plot does show brightening of the region. The emission gap coincides with the passage of the X-ray source. Additionally it may also coincide with the $\mathrm{X}$-line (current sheet) passage.

The Doppler velocity diagram is formed in the same way as in the previous part of the Fig. 4. However, this extended region has a strong red-shifted component along the bottom and the remaining part is weakly blue shifted. To interpret this it was important to have a clear understanding of the loops structure orientation.

\subsubsection{Magnetic field extrapolation}

Figure 10 illustrates the presence of a single bipolar structure on the 5th April. Over the following days, numerous bipoles emerge, resulting in a complex region of magnetic activity. The flare loops appeared to connect opposite polarity magnetic concentrations, belonging to different bipoles. The shape and orientation of the loops in this active region (AR) may be determined by producing a coronal field model and identifying the field lines that best match the observed loops. In this way we can go from the $2 \mathrm{D}$ view given by the observations to the $3 \mathrm{D}$ view provided by the model. These $3 \mathrm{D}$ models allow us to calculate the three components of the magnetic field vector: one along the line of sight and two on a plane orthogonal to it. From the line of sight field component, the direction of the magnentic field vector and line of sight velocity measurements, we can infer the direction of the plasma flow at each point along a loop (see Harra et al. 2004 and the last paragraph in this section).

At the disk centre the component of the field orthogonal to the photosphere $\left(B_{z}\right)$, which is the one used as a boundary condition in a magnetic field model, differs very little from the line of sight $\left(B_{l}\right)$ value. Unfortunately, moving away from this position, the measured line of sight magnetic field become less representative of $B_{z}$. Furthermore, the field becomes highly distorted through foreshortening in places where the field vector is inclined to the vertical, e.g. in penumbrae. In our case, AR 9901 is located very close to the limb (W69 N22) at the time of the event. However, we can proceed, as done by Harra et al. (2004), 

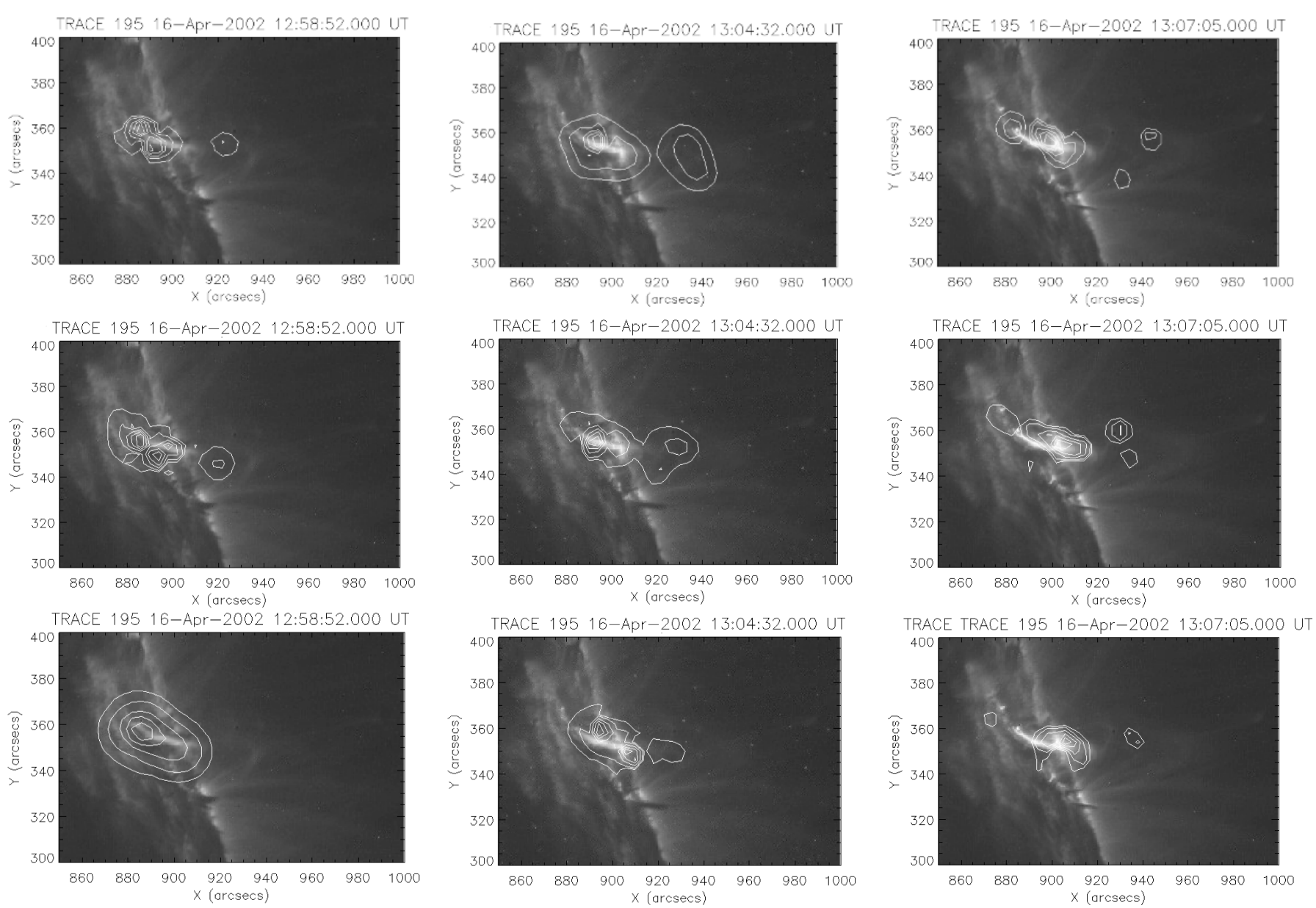

Fig. 6. The images in this figure are all TRACE $195 \AA$ A images. On these images are RHESSI contours at $80 \%, 60 \%, 40 \%, 20 \%$ and $10 \%$ of the peak intensity. The top row shows contours from the $5-10 \mathrm{keV}$, the second row shows $10-15 \mathrm{keV}$ and the final row has $15-20 \mathrm{keV}$ contours. Of particular note is the coronal source. One can see that this source moves with time from left to right, i.e. upward.

and produce a coronal field model using magnetic observations obtained some days before the arrival of the AR at the limb. We rotate the calculated field to the location of the AR at the appropriate time and compute the field lines that best fit the observed flare loops.

We selected a magnetogram from the Michelson Doppler Imager (MDI; Scherrer et al. 1995) taken on 14 April at 20:50 UT, 40 hours prior to this event and corrected the data assuming that the magnetic field vector is perpendicular to the photosphere. With these corrected data as boundary conditions, we computed a coronal field model under the linear (or constant $\alpha$ ) force-free field assumption, $\nabla \times \boldsymbol{B}=\alpha \boldsymbol{B}$, using a fast Fourier transform method, as proposed by Alissandrakis (1981).

To determine the value of the free parameter of the model $(\alpha)$ we used an EIT (Delaboudiniére et al. 1995) image, taken on 16 April, overlaid on a co-temporal MDI map to identify the location of the magnetic footpoints of the flare loops relative to the active region neutral line. After verifying that the orientation of the neutral line had not changed from April 14 to April 16 (using an MDI movie), we obtained the value of $\alpha=1.5 \times 10^{-2}$. With this value we could compute the field lines that, when rotated to the time of the flare, best match the EIT flare loops (whose shapes agree with those of the TRACE $195 \AA$ loops seen in Fig. 2)

The extrapolated field lines are shown in Fig. 11. From the view of the extrapolated loops on 14th April, it is clear that the southernmost footpoints are to the west, whereas the northern footpoints are more to the east. This is important information which would be impossible to determine from the TRACE images alone. It should be noted that although the extrapolated loop is not a perfect match with the flare loop it does show consistency with footpoint position. When we explored loop shapes with slightly different footpoint positions practically all computed loops had the same plasma flow direction. It is also worth note that the height of the loops plotted was multiplied by a factor of 2 to achieve a better match with the observations, but this does not influence the spatial orientation.

We can further relate the information of the magnetic field model to the CDS velocities to understand the direction of plasma flow along the observed structure, as done by Harra et al. (2004). Following the flux rope passage through the slit there is a time period where the emission is very low. During this time, the coronal X-ray source passes through the slit and probably also the $\mathrm{x}$-line. From about 13:10 UT we begin to see the cusp region, where the material has begun to cool 


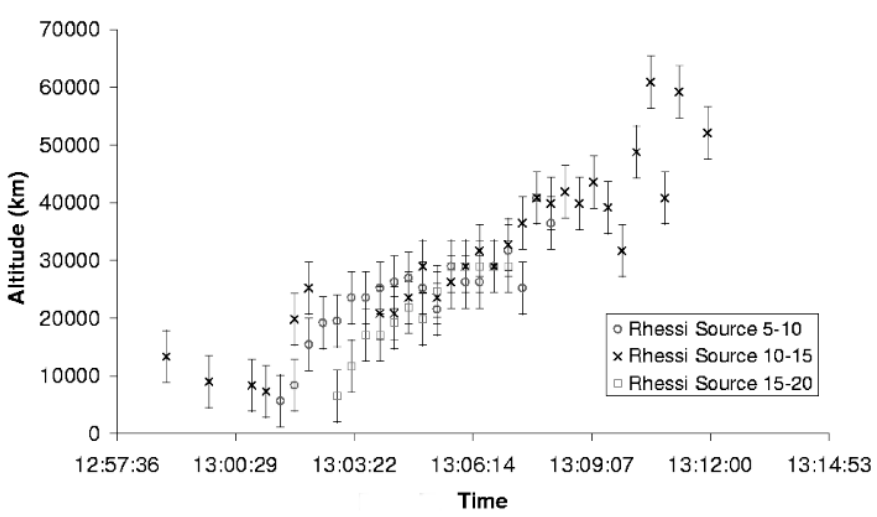

Fig. 7. This height-time plot is derived from the coronal HXR source over three separate energy bands.
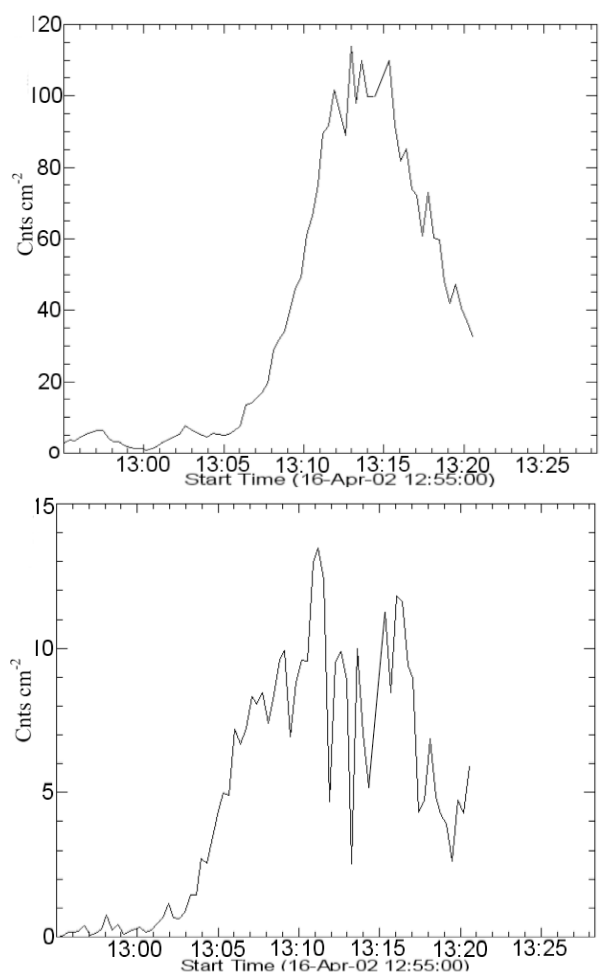

Fig. 8. The top light curve is constructed to show how the number of counts changes with time for the loop top source and the loop footpoints. They have been grouped together as it is very difficult to distinguish between them accurately. The bottom light curve is that of the coronal X-ray source located above loop top source.

back into the passband. This is much hotter than observed by TRACE $195 \AA$, therefore it is not unexpected that these loops are not seen at the position of the slit. However, the material is beginning to cool as it has come back into view and is likely to continue to fall. If one takes the extrapolations to be a representation of the loop orientation, then down-flows in the northern half of the loop would have a blue-shifted velocity and conversely the southern half would be red-shifted. We also see enhancement of the blue-shifted values in the north. However, due to line of sight effects the red-shifted region can be foreshortened. Additionally low velocities are expected at the top of the loops. Figure 4 shows a large grey region in the middle,

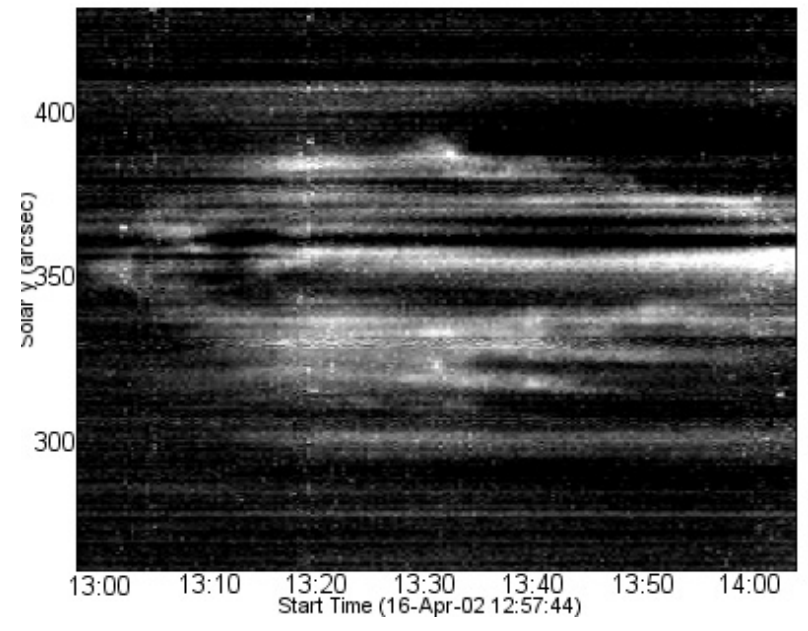

Fig. 9. A synthetic slit image assembled from the TRACE data cube, designed to mimic the intensity data gathered from CDS.

possibly where the loop is almost perpendicular to the line of sight.

With the assumption that the velocities measured in CDS at both sides of the central region are mainly due to plasma flows along field lines whose shape and direction are similar to that of our extrapolated loops. We can combine the direction of the line of sight velocity with that of the $B_{l}$ (line of sight magnetic field component) along the legs of the computed loops with the vector magnetic field (see Eq. (1) in Harra et al. 2004) and attempt to determine the direction of the plasma flow. From the extrapolation we find that $B_{l}$ points towards the observer along both legs of the computed loops, while the line of sight velocity component $\left(v_{l}\right)$ points towards the observer in the north and away in the south. The plasma appears to be flowing down along both sides of the observed structure compared to the direction of the field given by the arrows on the lines in Fig. 11. Therefore, we suggest that hot material is falling along the newly reconnected loops as it cools.

\subsection{The CME}

The observed flux rope, previously seen in the low corona, is later seen as part of a CME in LASCO (Fig. 12). CMEs related to filament eruptions are thought to have a three-part structure (Illing \& Hundhausen 1985), indicating the initial leading edge seen in LASCO C2 is not representative of the leading edge in the TRACE $195 \AA$ movies.

The three-part structure consists of a bright front, followed closely by a cavity thought to be the magnetic structure of a flux rope and then a bright core region thought to be the filament/prominence material it contains. Accepting our initial observations in TRACE $195 \AA$ are of the flux rope, it is, therefore, not sufficient to simply measure the leading edge of the CME again in LASCO.

The CME we observe is very slow and weak. It is impossible to distinguish a clear three-part structure. We decided to measure both the leading edge of the CME and also the trailing edge through the use of difference images. The front of a bright 


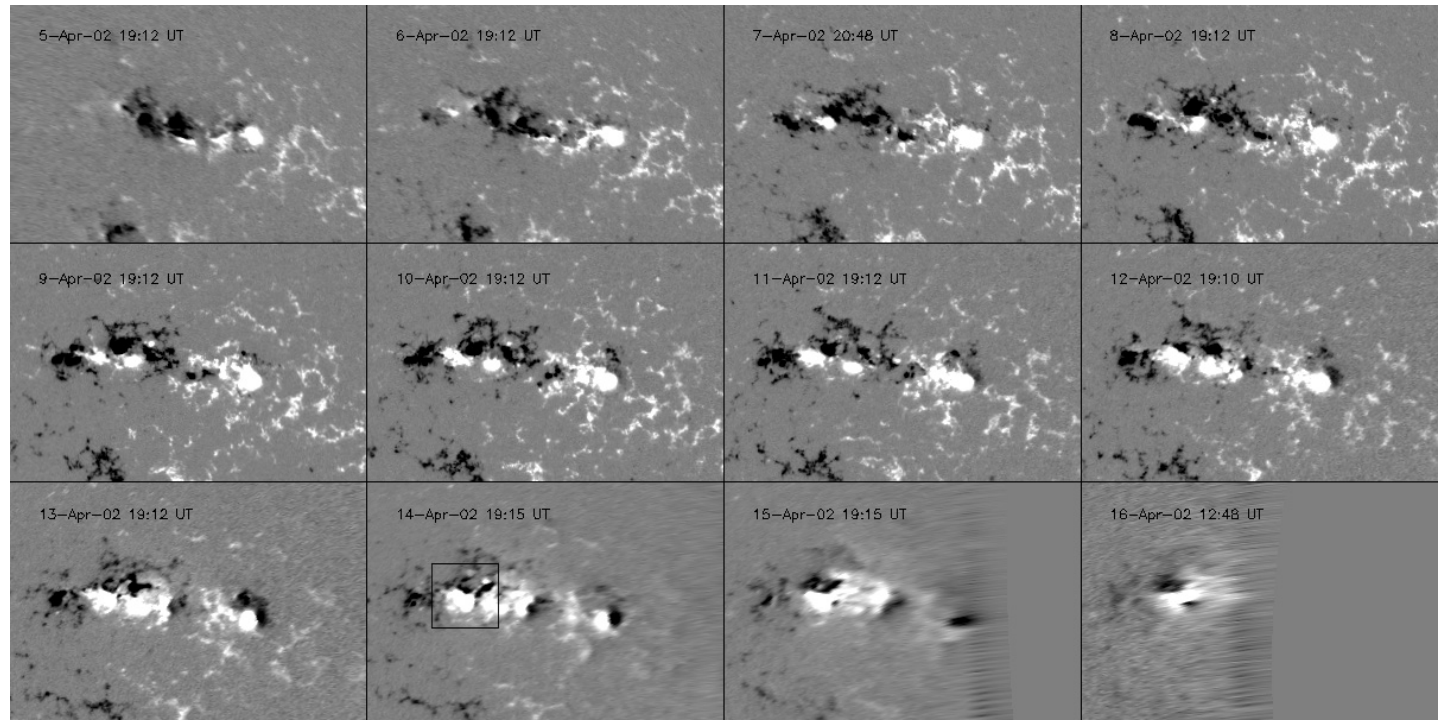

Fig. 10. A time series of MDI magnetograms, rotated to central meridian position in the days running up to the event on 16 th April 2002. One can see the evolution of the region from the top left to the bottom right where projection effects distort the image dramatically. In the image from 14th April a box is drawn representing the region used in the extrapolations of Fig.11.

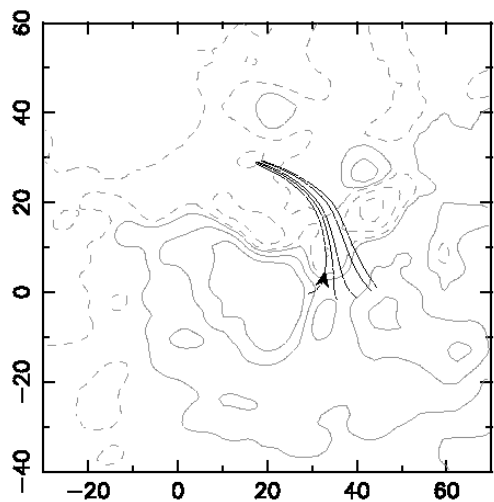

(a)

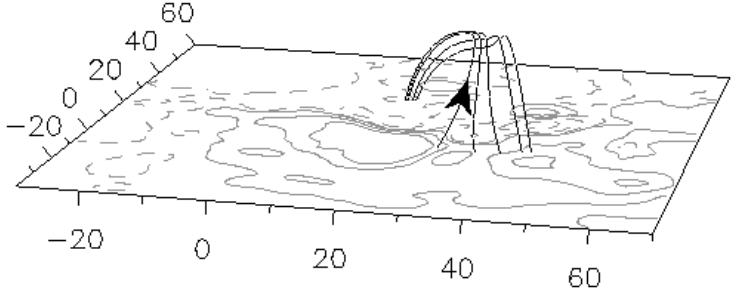

(b)

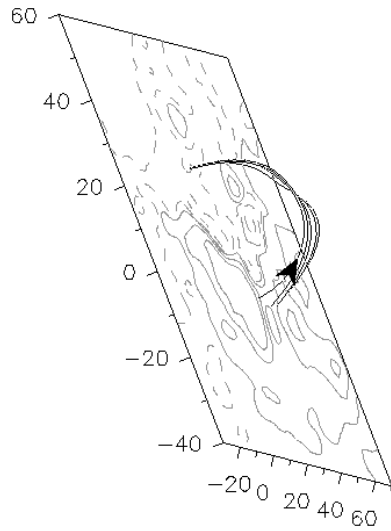

(c)

Fig. 11. a) Magnetic field lines extrapolated (local field of view, as if the AR is located at disk centre) in the linear force-free approximation $\left(\alpha=0.015 \mathrm{Mm}^{-1}\right)$ using a SOHO/MDI full-disc magnetic map as boundary condition two days prior to the event. b) As in a) in a different point of view to enable one to see the arcade orientation. c) As in a) with the AR rotated to the time of the event. The axes are measured in Mm and the field of view extends from 290" to $428^{\prime \prime}$ in the North-South direction and from 838" to 977" in the East-West direction (see Fig. 2). The isocontour values are $\pm 100,500,1000 \mathrm{G}$.

region signifies the leading edge and the front of the dark trailing region has been classified as the rear. This provides outer boundaries for the location of the previously mentioned flux rope.

With these additional points (Fig. 13), obtained from LASCO C2, it becomes obvious that simple linear fits are not appropriate. It is impossible for a linear fit to be consistent with these combined observations as the required altitudes cannot be reached in the time available. Instead some other fit was required to account for this rapid increase in altitude. Several samples of fits are shown in Fig. 13. An exponential fit is most plausible, which rules out a constant acceleration process. This fits both the TRACE data section of the plot and also allows the heights measured in LASCO to be achieved. This is consistent with the results of Gallagher et al. (2003) who had a similar plot for the 21st April 2002 CME. Note that their LASCO measurements were leading edge based, possibly giving a deceptively high value. However, an exponential rise is required for their dataset. In this event an exponential fit for the rapid rising phase and a linear fit for the LASCO section, results in a good fit with a high $R^{2}$ statistic (0.99 in the growth section and 0.96 in the linear region). When combined, we can differentiate these to give a velocity profile and an acceleration profile as seen in Fig. 14. The flux rope clearly slows down following the peak as throughout the linear phase the velocity remains constant at $135 \mathrm{~km} \mathrm{~s}^{-1}$. Although a dashed line from the end of the TRACE data to the beginning of the C2 observations is plotted, we have not differentiated. 


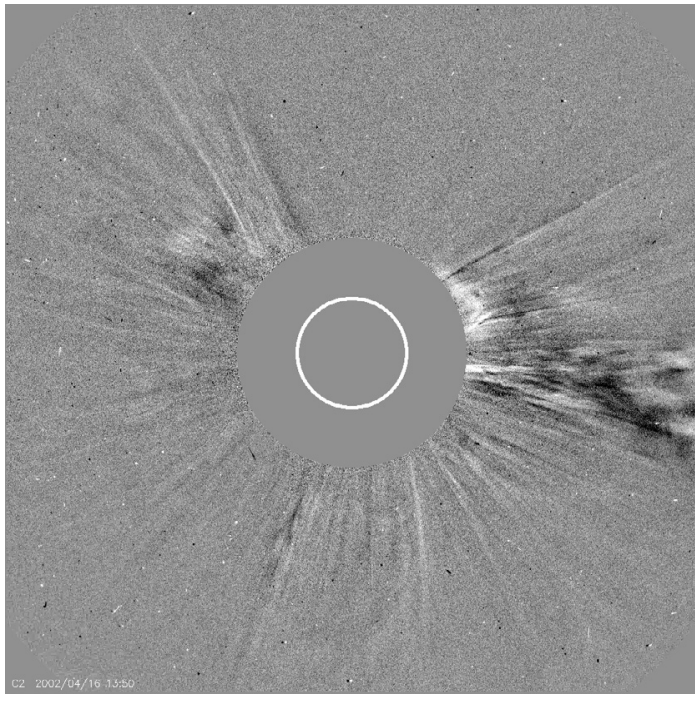

Fig. 12. LASCO C2 difference image of the CME on 16th April 2002 at 13:50 UT, where the CME can be seen emerging from the occulting disk in the northwest.

\section{Discussion}

We have summarised our findings in a diagram as shown in Fig. 15. Based on a model suggested by Shibata et al. (1995), however, we have added the velocity information for the event studied above. The diagram shows the lifting flux rope, below which is the current sheet where reconnection occurs, and finally beneath this are the cooler flare loops. Traditionally at the footpoints of these flare loops there is hard X-ray emission from the collision of high-energy electrons impacting the dense chromosphere. However, in this event, footpoint emission is much lower than expected due to the unusually dense loop top.

The lifting flux rope was seen in TRACE $195 \AA$ A (Fig. 3) and CDS Fe XIX, followed by the CME in LASCO C2. The reason for this lift off is unclear and many models attempt to explain it (e.g. Forbes 2000; Török et al. 2004). With careful study of the TRACE $195 \AA$ movie it is apparent that there is some global deformation of the flux rope system although quantitative measurement of this is difficult. As it rises it crosses the CDS slit field of view (Fig. 4). The Doppler velocities from the same figure indicate that there is an inherent helical structure of the loop, highlighted by helical flows. A similar observation was made for an on-disk flux rope by Foley et al. (2001) and Pike $\&$ Mason (2002). They found that there was significant blue shift attributed to the rising flux rope and a red shifted component, either attributed to an untwisting of the flux rope or to falling material. In the case of the 16th April 2002 red and blue shifted emissions were found on opposing sides.

From Fig. 15, as the flux rope begins its lift-off phase, it would drag magnetic field lines with it. This would stretch the field beneath it forming a current sheet. At this point magnetic reconnection can begin and so too can the flare, also, reconnection jets would be produced. The RHESSI observations of this event show a loop top source and weak footpoints. The loop top source is unusually bright relative to the footpoints. Assuming that this event is homologous with these of the previous two

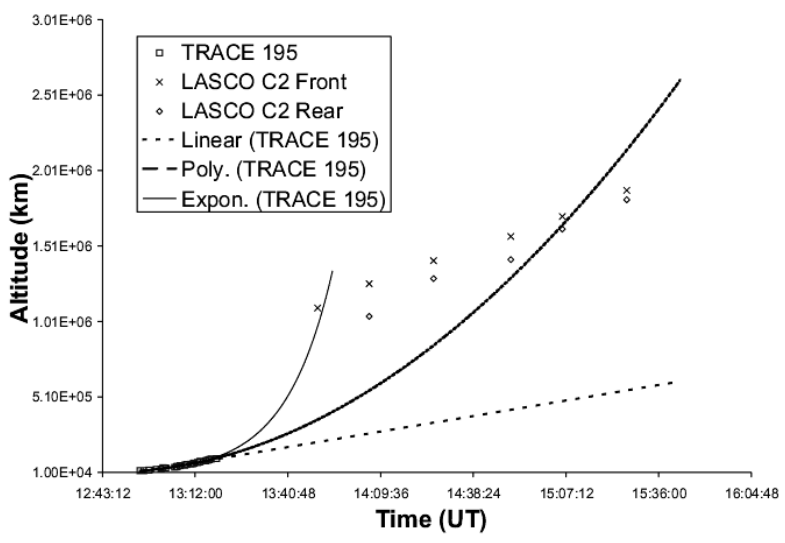

Fig. 13. Height-time plot for the lifting flux rope as seen by TRACE and LASCO C2, with three different types of fit. The crosses represent the leading and trailing edge of the LASCO CME. We have an exponential growth, which can be seen to meet the required height in the required time. Then there is the polynomial fit and the linear fit which can never reach the required heights.

days, then it is described as a loop which is collisionally thick at electron energies up to $50 \mathrm{keV}$ (Veronig \& Brown 2004). There is a coronal source observed up to $25 \mathrm{keV}$, and shown in Fig. 6. This source moves upward and follows the rising flux rope with a comparable velocity to that of the flux rope, implying an intimate link. We suggest that this indicates the formation of a plasmoid as observed by Tsuneta (1997). This source was tracked by plotting the brightest point location and can be seen in Fig. 5.

From Fig. 4 we see that following the passage of the flux rope there is a period of weak emission. This corresponds to the passage of the coronal X-ray source and may also correspond to the magnetic $\mathrm{x}$-line or current sheet. When one studies the TRACE $195 \AA$ movie closely, the brightening of loops and the formation of a cusp can be seen, the peak of which is located around the position of the CDS slit at the time of the gap. At this point the emission measure would be low and little emission would be expected. This is also apparent if one studies the stack plot from TRACE in Fig. 9. Even here there is little emission in that location and time period.

Following this time there is an extended period of about $90 \mathrm{~min}$, when the velocity pattern suggestive of down-flows, along the newly reconnected loops is observed. The Doppler velocities seen by CDS in Fig. 4 suggest that when taking into account the orientation of the loop determined through magnetic extrapolation (Fig. 11) we are seeing the falling material either coming towards or moving away from us. We believe that observations represent this rather than general loop shrinkage as the largest Doppler shifts are seen to be at the edges of this expanded region with much lower velocities in between. However, it is very difficult to distinguish between these two mechanisms with the available data. It may be that this grey region represents the global shrinkage and the bright edges are from the loop leg down-flows.

The flux rope continues to rise and is observed as a CME in LASCO C2. The leading edge was measured along with the rear of the CME and the two lines were plotted on the 

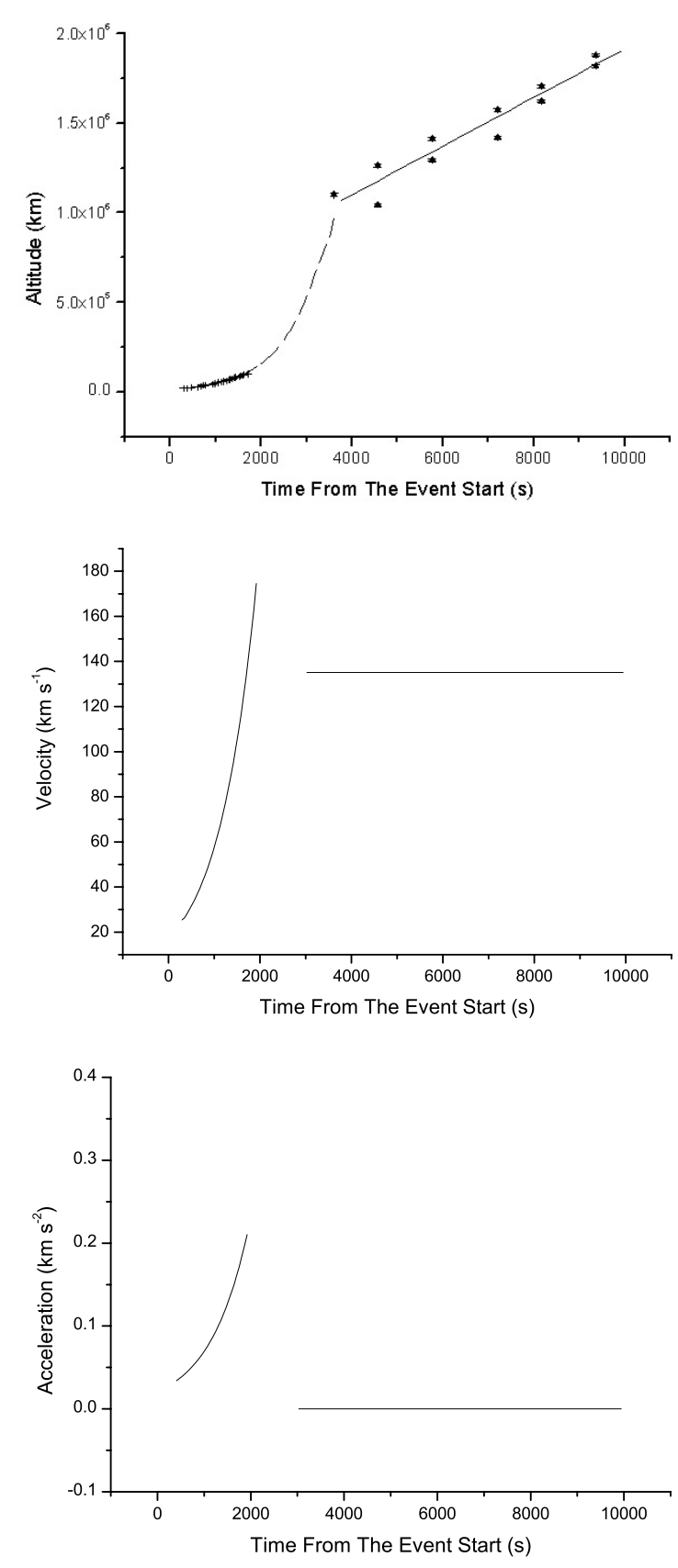

Fig. 14. Height-time plot for the lifting flux rope as seen by TRACE and LASCO C2 with error bars representing five pixels. The dashed line represents the continuation of the exponential fit to the TRACE data. This is followed by the velocity profile and acceleration profile derived from the first and second derivative of the fits to the data.

height-time plot, which can be seen in Fig. 14. It is clear that a massive velocity change would be required in order to reach the $\mathrm{C} 2$ heights in the available time. Only the exponential function can provide this. This exponential growth is present in ideal instabilities such as the kink instability simulations by Török et al. (2004) and Kliem et al. (2004). These authors suggest the kink instability of a coronal flux rope as a possible initiation mechanism of solar eruptions. Our data suggests that the CME has either reached a steady state by the time it is observed by

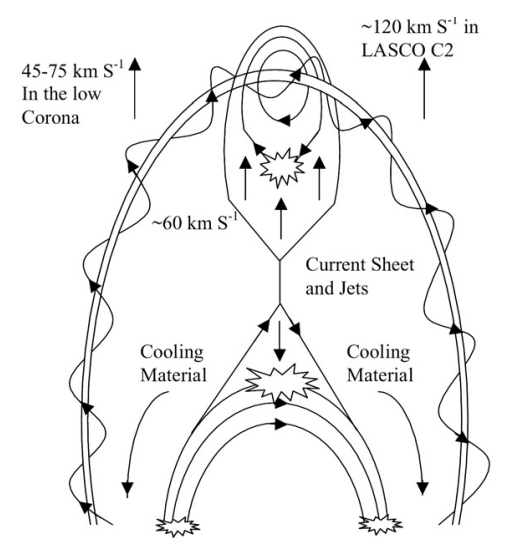

Fig. 15. Cartoon representing the entire event, based upon our observations from all instruments.

LASCO or is slowing down. Gallagher et al. (2003) presented an event with a very similar profile with additional data from UVCS which provided a valuable point between TRACE and LASCO. Even without this we are able to show that an exponential function is required to produce the altitudes required in the allotted time frame.

The fit that we produced could also be differentiated to give velocity and acceleration profiles. We can see that this event falls in the impulsive acceleration category defined by Zhang et al. (2004). Much like their event, it can be described in a three-phase scenario. First there is the initiation phase, characterised by the slow rise in the corona. In our case this corresponds to the TRACE $195 \AA$ observations. We were able to measure this over some $24 \mathrm{~min}$ with a maximum velocity of $\sim 100 \mathrm{~km} \mathrm{~s}^{-1}$. Throughout this time period the GOES light curve begins to rise to a peak at $\sim 13: 25 \mathrm{UT}$.

The second phase, characterised by a rapid increase in velocity, is the most problematic for us to profile. We have a data gap throughout this phase. However, using the LASCO data and the end of the TRACE data as limits we are confident there is a rapid velocity increase during this time. The rising filament actually speeds up by a factor of 1.7 at the start of the impulsive energy release and as our fit shows must go through further acceleration before reaching 1.5 solar radii.

The third and final phase is merely a propagation phase with little or no acceleration. Our case indicates that the acceleration falls to zero, as suggested. A linear fit describes the final stages indicating the velocity drops to a mere $\sim 135 \mathrm{~km} \mathrm{~s}^{-1}$.

It is noteworthy, however, that our second and third phases both correspond to the decay phase on the GOES light curve. This is in contrast to the work of Zhang et al. (2004), in which the second phase corresponded to a rapid increase in GOES flux. We have demonstrated that the X-ray emission rise is coincident in time with the CME initiation phase, which suggests a physical link.

\section{Conclusions}

We have seen that the 16th April 2004 event is associated with a flux rope (filament) lift, forming a CME. This CME has a height-time profile that requires an exponential fit to 
achieve the observed altitudes in the given time. This exponential growth suggests that the acceleration is caused by some kind of instability, which may be attributed to the kink instability in the rising flux rope.

As the flux rope / filament lifts it is seen to have helical flows, suggesting a helical magnetic structure. This is seen to be the case through Doppler measurements with CDS.

There were also observations from RHESSI which show the loop top source much brighter than the footpoint sources as similar to the ones first observed by Veronig \& Brown (2004). In addition, however, there was a coronal source that appears to follow the rising flux rope, and is above the top of the cusp with a velocity comparable to that of the rising flux rope. It is likely that we are seeing the RHESSI manifestation of the plasmoid observed by Tsuneta (1997). This source passes through the CDS slit although it cannot be seen in the Fe XIX passband, as it is almost certainly at too high a temperature.

Additionally from magnetic extrapolations we have determined that the flux rope lifts mostly in a westward direction with an offset towards our line of sight. From study of the CDS Dopplergrams we have also seen that in the cusp region under the expected location of the current sheet there are likely to be down flows along the newly reconnected loops. These probably contain hot material, which is cooling and falling back towards the surface.

Acknowledgements. The authors thank the TRACE, RHESSI, $\mathrm{SOHO} / \mathrm{CDS}, \mathrm{LASCO} / \mathrm{C} 2$ and MDI consortia as well as the Mullard Space Science Laboratory Solar UK Research Facility (SURF) for the data. SOHO is a joint project by ESA and NASA. They also wish to thank T. Török and B. Kliem for inspiring discussion. In addition we thank P. Démoulin and J. L. Culhane for critical reading of the manuscript. C.P.G. is grateful to PPARC for postgraduate student funding. L.vD.G. is supported by the Hungarian Government grant OTKA T-038013. C.H.M. and L.vD.G. thank TET (Hungary) and SECyT (Argentina) for financial support through their cooperation program (HU/A01/UIII/01 and AR03/02). C.H.M. is grateful for an MSSL/UCL visitor's grant. C.H.M. thanks for support from the Argentinean grants UBACyT X329, CONICET PIP-2388 and ANPCYT PICT-12187.

\section{References}

Alissandrakis, C. E. 1981, A\&A, 100, 197

Antiochos, S. K., DeVore, C. R., \& Klimchuck, J. A. 1999, ApJ, 510, 485

Brueckner, G. E., Howard, R. A., Koomen, M. J., et al. 1995, Sol. Phys., 162, 357

Delaboudiniére, J.-P., Artzner, G. E., Brunaud, J., et al. 1995, Sol. Phys., 162, 291

Foley, C. R., Harra, L. K., Culhane, et al. 2001, ApJ, 560, L91

Forbes, T. G. 2000, J. Geophys. Res., 105, 153

Forbes, T. G. 2003, Adv. Space Res., 32, 1043

Gallagher, P. T., Dennis, B. R., Krucker, A. K., et al. 2002, Sol. Phys., 210, 341

Gallagher, P. T., Lawrence, G. R., \& Dennis, B. R. 2003, ApJ, 588, L53

Handy, B. N., Acton, L. W., Kankelborg, C. C., et al. 1999, Sol. Phys., 187,229

Harra, L. K., Mandrini, C. H., \& Matthews, S. A. 2004, Sol. Phys., in press

Harrison, R. A., Sawyer, M. K., Carter, M. K., et al. 1995, Sol. Phys., 162,233

Illing, R. M. E., \& Hundhausen, A. J. 1985, J. Geophys. Res., 90, 275

Kliem, B., Titov, V. S., \& Török, T. 2004, A\&A, 413, L23

Klimchuk, J. A. 2000, AGU Monograph Series, ed. P. Song, G. Siscoe, et al. (Washington: Agu), 143

Kohl, J. L., Esser, R., Gardner, L. D., et al. 1995, Sol. Phys., 162, 313

Lin, R. P., Dennis, B. R., Hurford, G. J., et al. 2002, Sol. Phys., 210, 3

Metcalf, T. R., Hudson, H. S., Kosugi, et al. 1996, ApJ, 466, 585

Pike, C. D., \& Mason, H. E. 2002, Sol. Phys., 206, 359

Scherrer, P. H., Bogart, R. S., \& Bush, R. I. 1995, Sol. Phys., 162, 129

Shibata, K., Masuda, S., Shimojo, M., et al. 1995, ApJ, 451, L83

St. Cyr, O. C., Howard, R. A., Simnett, G. M., et al. 1997, Proc. 31st ESLAB Symp, Correlated Phenomena at the Sun, in the Heliosphere and in Geospace

Sui, L., Holman, G., \& Dennis, B. 2004, ApJ, 612, 546

Török, T., Kliem, B., \& Titov, V. S. 2004, A\&A, 413, L27

Tsuneta, S. 1997, ApJ, 483, 507

Veronig, A. M., \& Brown, J. C. 2004, ApJ, 603, L117

Zhang, J., Dere, K. P., Howard, R. A., et al. 2004, ApJ, 604, 420 